\title{
CHARACTERIZING THE DEPOLARIZING QUANTUM CHANNEL IN TERMS OF RIEMANNIAN GEOMETRY
}

\author{
CARLO CAFARO $^{1}$ and STEFANO MANCINI ${ }^{1,2}$ \\ ${ }^{1}$ School of Science and Technology, Physics Division, \\ University of Camerino, I-62032 Camerino, Italy \\ ${ }^{2}$ INFN, Sezione di Perugia, I-06123 Perugia, Italy
}

\begin{abstract}
We explore the conceptual usefulness of Riemannian geometric tools induced by the statistical concept of distinguishability in quantifying the effect of a depolarizing channel on quantum states. Specifically, we compare the geometries of the interior of undeformed and deformed Bloch spheres related to density operators on a two-dimensional Hilbert space. We show that randomization emerges geometrically through a smaller infinitesimal quantum line element on the deformed Bloch sphere while the uniform contraction manifests itself via a deformed set of geodesics where the spacial components of the deformed four-Bloch vector are simply the contracted versions of the undeformed Bloch vector components.
\end{abstract}

PACS numbers: Riemannian Geometry (02.40.Ky), Quantum Information (03.67.-a), Open Quantum Systems $(03.65 . \mathrm{Yz})$.

\section{INTRODUCTION}

It is well-accepted in the scientific community that geometry plays an important role in characterizing and understanding both classical and quantum physics. As a matter of fact, it has been an old dream to reduce the fundamental laws of physics to geometry since Einstein's formulation of general relativity. In particular, it is a remarkable achievement that all the building blocks of quantum field theory can be formulated in terms of geometric concepts such as vector bundles, connections, curvatures, covariant derivatives and spinors [1]. More recently, Marmo and coworkers have pointed out the potential usefulness of a geometrical formulation of quantum theory to investigate the entanglement and separability for quantum states describing composite systems [2].

In 1985, Campbell showed that geometry can be introduced into probability calculus as follows [3] : for a fixed probability distribution, define the inner product of two random variables to be the expectation of the product of these variables. Differential geometry emerges when we consider varying the probability distribution, either directly or through changing parameters on which the distribution depends. Within such a geometric framework, the sets of probability distributions are viewed as differentiable manifolds, the random variables appear as vectors and the expectation values of random variables are replaced with inner products in tangent spaces to such manifolds of probabilities. In 1995, Braunstein and Caves extended Campbell's ideas to the quantum framework [4].

Here, inspired by Marmo and following the lead of Braunstein and Caves, we explore the possibility of the conceptual usefulness of differential geometric tools in quantifying the effect of depolarizing channels on quantum states by comparing the geometries of the interior of the undeformed and deformed Bloch spheres related to density operators on a two-dimensional Hilbert space.

\section{DIFFERENTIAL GEOMETRY OF DENSITY OPERATORS}

For a more detailed presentation of this preliminary material, we refer to [4, 5]. Consider the quantum analogue $\mathcal{M}_{\vec{\rho}}$ of the probability simplex, the space of density operators $\vec{\rho}$ written as vectors in $\mathcal{L}(\mathcal{H})$, the linear space of all linear operators on a $n$-dimensional Hilbert space $\mathcal{H}$,

$$
\mathcal{M}_{\vec{\rho}} \stackrel{\text { def }}{=}\left\{\vec{\rho} \in \mathcal{L}(\mathcal{H}): \vec{\rho} \stackrel{\text { def }}{=} \sum_{i, j=1}^{n} \rho^{i j} \vec{e}_{i j}, \vec{\rho}=\vec{\rho}^{\dagger}, \operatorname{tr}(\vec{\rho})=1, \vec{\rho} \geq 0\right\} .
$$

The space $\mathcal{M}_{\vec{\rho}}$ is an $\left(n^{2}-1\right)$-dimensional real manifold with complicated boundary. An arbitrary linear operator vector $\vec{V}$ on $\mathcal{H}$ can be decomposed in terms of an operator vector basis $\vec{e}_{i j} \stackrel{\text { def }}{=}|i\rangle\langle j|$ with $i, j=1, \ldots, n$ as follows,

$$
\vec{V}=\sum_{i, j=1}^{n}\langle i|\vec{V}| j\rangle \vec{e}_{i j}=\sum_{i, j=1}^{n} V^{i j} \vec{e}_{i j}
$$


The tangent space at $\vec{\rho}$ is an $\left(n^{2}-1\right)$-dimensional real vector space of traceless Hermitian operators $\vec{T}$,

$$
\vec{T}=\sum_{i, j=1}^{n} T^{i j} \vec{e}_{i j}, \operatorname{tr}(\vec{T})=0
$$

The action of 1 -forms $\tilde{F}$ expanded in terms of the dual basis $\tilde{\omega}^{j i} \stackrel{\text { def }}{=}|i\rangle\langle j|$,

$$
\tilde{F} \stackrel{\text { def }}{=} \sum_{i, j=1}^{n} F_{i j} \tilde{\omega}^{j i}
$$

on density operators $\vec{\rho}$ is defined as follows,

$$
\tilde{F}(\vec{\rho}) \equiv\langle\tilde{F}, \vec{\rho}\rangle=\sum_{i, j, l, k=1}^{n} F_{i j} \rho^{l k}\left\langle\tilde{\omega}^{j i}, \vec{e}_{l k}\right\rangle=\sum_{i, j, l, k=1}^{n} F_{i j} \rho^{l k} \delta_{l}^{j} \delta_{k}^{i}=\sum_{i, j=1}^{n} F_{i j} \rho^{j i}=\operatorname{tr}(\tilde{F} \vec{\rho}) \equiv\langle\tilde{F}\rangle .
$$

Therefore, an Hermitian 1-form $\tilde{F}=\tilde{F}^{\dagger}$ is an ordinary quantum observable with $\langle\tilde{F}, \vec{\rho}\rangle=\langle\tilde{F}\rangle$. A metric structure $\mathbf{g}_{\vec{\rho}}(\cdot, \cdot)$ on the manifold $\mathcal{M}_{\vec{\rho}}$ can be introduced by defining the metric's action on a pair of 1 -forms $\tilde{A}$ and $\tilde{B}$ as follows,

$$
\mathbf{g}_{\vec{\rho}}(\tilde{A}, \tilde{B}) \stackrel{\operatorname{def}}{=}\left\langle\frac{\tilde{A} \tilde{B}+\tilde{B} \tilde{A}}{2}\right\rangle=\operatorname{tr}\left[\left(\frac{\tilde{A} \tilde{B}+\tilde{B} \tilde{A}}{2}\right) \vec{\rho}\right]=\operatorname{tr}\left[\frac{\tilde{A}}{2}(\vec{\rho} \tilde{B}+\tilde{B} \vec{\rho})\right]=\left\langle\tilde{A}, \mathcal{R}_{\vec{\rho}}(\tilde{B})\right\rangle
$$

where $\mathcal{R}_{\vec{\rho}}(\tilde{B})$ is the raising operator mapping 1-forms (lower covariant components) to vectors (upper contravariant components),

$$
\mathcal{R}_{\vec{\rho}}(\tilde{B}) \stackrel{\text { def }}{=} \frac{\vec{\rho} \tilde{B}+\tilde{B} \vec{\rho}}{2}
$$

Such a metric is formulated in terms of statistical correlations of quantum observables. Using the lowering operator $\mathcal{L}_{\vec{\rho}}(\vec{A})$ that maps vectors to 1 -forms,

$$
\mathcal{L}_{\vec{\rho}}(\vec{A})=\mathcal{R}_{\vec{\rho}}^{-1}(\vec{A})
$$

we can also define the action of the metric tensor $g_{\vec{\rho}}(\cdot, \cdot)$ on a pair of vectors $\vec{A}$ and $\vec{B}$,

$$
\mathbf{g}_{\vec{\rho}}(\vec{A}, \vec{B}) \stackrel{\text { def }}{=}\left\langle\mathcal{L}_{\vec{\rho}}(\vec{A}), \vec{B}\right\rangle=\operatorname{tr}\left[\vec{B} \mathcal{L}_{\vec{\rho}}(\vec{A})\right]
$$

The quantum line element $d s^{2}=\mathbf{g}_{\vec{\rho}}(d \vec{\rho}, d \vec{\rho})$ with $d \vec{\rho}$ given by,

$$
d \vec{\rho}=\sum_{j=1}^{n} d p^{j}|j\rangle\left\langle j\left|+i d \theta \sum_{m, l=1}^{n}\left(p^{m}-p^{l}\right) h_{l m}\right| l\right\rangle\langle m|
$$

and with $e^{i d \theta h}$ an infinitesimal unitary transformation on the orthonormal basis that diagonalizes $\vec{\rho}$, reads

$$
d s^{2}=\mathbf{g}_{\vec{\rho}}(d \vec{\rho}, d \vec{\rho}) \stackrel{\text { def }}{=} \operatorname{Tr}\left[d \vec{\rho} \mathcal{L}_{\vec{\rho}}(d \vec{\rho})\right]=\sum_{k=1}^{n} \frac{\left(d p^{k}\right)^{2}}{p^{k}}+2 d \theta^{2} \sum_{j \neq k} \frac{\left(p^{j}-p^{k}\right)^{2}}{\left(p^{j}+p^{k}\right)}\left|h_{j k}\right|^{2} .
$$

Notice that the above quantum line element is identical to the distinguishability metric for density operators obtained in [6] by optimizing over all generalized quantum measurements for distinguishing among neighboring quantum states.

\section{DEPOLARIZED DENSITY OPERATORS: THE CONVENTIONAL APPROACH}

The two-dimensional depolarizing channel is an error model which can be described as follows [7]: this channel, with probability $1-p$, passes a qubit without altering its state; with probability $p$, an error of the Pauli-type occurs 
(application of one among the equally likely Pauli errors $\sigma_{1}, \sigma_{2}, \sigma_{3}$ ). In terms of the Kraus operator-sum decomposition of the depolarizing channel, it turns out that an arbitrary initial density operator $\rho$ of the qubit is mapped into,

$$
\rho \rightarrow \rho^{\prime}=(1-p) \rho+\frac{p}{3}\left(\sigma_{1} \rho \sigma_{1}+\sigma_{2} \rho \sigma_{2}+\sigma_{3} \rho \sigma_{3}\right)
$$

An alternative manner to characterize the action of a depolarizing channel on quantum states can be described by assuming that the initial state is one of the following four mutually orthogonal maximally entangled two-qubits states,

$$
\left|\psi^{ \pm}\right\rangle \stackrel{\text { def }}{=} \frac{1}{\sqrt{2}}\left[|01\rangle_{A B} \pm|10\rangle_{A B}\right] \text { and, }\left|\phi^{ \pm}\right\rangle_{A B} \stackrel{\text { def }}{=} \frac{1}{\sqrt{2}}\left[|00\rangle_{A B} \pm|11\rangle_{A B}\right]
$$

For instance, take into consideration $\left|\phi^{+}\right\rangle_{A B}$ and consider the action of the depolarizing channel on the first qubit,

$$
\rho_{\phi^{+}} \stackrel{\text { def }}{=}\left|\phi^{+}\right\rangle\left\langle\phi^{+}\right| \rightarrow \rho_{\phi^{+}}^{\prime}=\frac{4}{3} p\left(\frac{1}{4} I^{A B}\right)+\left(1-\frac{4}{3} p\right) \rho_{\phi^{+}} .
$$

Observe that $I^{A B}$ is the identity operator on the Hilbert space $\mathcal{H}_{A} \otimes \mathcal{H}_{B}$ and equals $I_{A B}=\rho_{\phi^{+}}+\rho_{\phi^{-}}+\rho_{\psi^{+}}+\rho_{\psi^{-}}$, with

$\rho_{\psi^{ \pm}} \stackrel{\text { def }}{=}\left|\psi^{ \pm}\right\rangle\left\langle\psi^{ \pm}\left|, \rho_{\phi^{ \pm}} \stackrel{\text { def }}{=}\right| \phi^{ \pm}\right\rangle\left\langle\phi^{ \pm}\right|$. From (14), it follows that a depolarizing error occurs with probability $\frac{4}{3} p$ and the error completely randomizes the state $\left|\phi^{+}\right\rangle_{A B}$ provided that $p \leq \frac{3}{4}$. The transformed density matrix $\rho_{\phi^{+}}^{\prime}$ becomes maximally random $\left(\rho_{\phi^{+}}^{\prime}=\frac{1}{4} I_{A B}\right)$ in the limiting case of $p=\frac{3}{4}$.

An additional manner to characterize depolarizing errors on density operators is that of considering its action on the Bloch sphere representation of an arbitrary initial density operator $\rho$ of the qubit,

$$
\rho=\frac{1}{2}(I+\mathbf{P} \cdot \boldsymbol{\sigma})
$$

where $\mathbf{P} \stackrel{\text { def }}{=} \operatorname{Tr}(\boldsymbol{\sigma} \rho)$ is the Bloch polarization vector. For $\|\mathbf{P}\|=1$ the density matrices describe a pure state whereas for $\|\mathbf{P}\|<1$ one has a mixed state. Thus, the density matrix $\rho$ is uniquely determined by a point of the unit sphere $0 \leq\|\mathbf{P}\| \leq 1$ (unit 3-ball). For depolarizing errors, the Bloch sphere contracts uniformly under the action of the depolarizing channel since the spin polarization of the qubit $\mathbf{P}$ is reduced by a factor $\left(1-\frac{4}{3} p\right)$ where $p$ denotes the error probability,

$$
\rho=\frac{1}{2}(I+\mathbf{P} \cdot \boldsymbol{\sigma}) \rightarrow \rho^{\prime}=\frac{1}{2}\left(I+\mathbf{P}^{\prime} \cdot \boldsymbol{\sigma}\right) \text { with } \mathbf{P}^{\prime}=\left(1-\frac{4}{3} p\right) \mathbf{P} .
$$

In summary, the two main features that characterize the action of the depolarizing channel on density operators is the randomization of maximally entangled quantum states (Eq. (14)) and the uniform contraction of the deformed Bloch sphere (Eq. (16) ). In which manner do these features emerge in a Riemannian geometric characterization of depolarizing channels? We attempt to provide an answer to this question in the next Section.

\section{DEPOLARIZED DENSITY OPERATORS: THE GEOMETRIC APPROACH}

Returning to the formalism introduced in Section II, it follows that an arbitrary density operator $\vec{\rho}$ reads,

$$
\vec{\rho}=\frac{1}{2}[\vec{I}+\mathbf{P} \cdot \overrightarrow{\boldsymbol{\sigma}}]=\frac{1}{2}[\vec{I}+\|\mathbf{P}\| \mathbf{n} \cdot \overrightarrow{\boldsymbol{\sigma}}]
$$

where the Bloch vector $\mathbf{P}$ and the Pauli (operator) vector $\overrightarrow{\boldsymbol{\sigma}}$ are,

$$
\mathbf{P} \stackrel{\text { def }}{=} \sum_{k=1}^{3} P^{k} \mathbf{e}_{k}=\|\mathbf{P}\| \mathbf{n} \text { and, } \overrightarrow{\boldsymbol{\sigma}} \stackrel{\text { def }}{=} \sum_{k=1}^{3} \vec{\sigma}^{k} \mathbf{e}_{k}
$$

respectively and where $\mathbf{e}_{k}$ are unit orthonormal vectors spanning $\mathbb{R}^{3}$. Recall that the infinitesimal quantum line element $d s^{2}$ is given by,

$$
d s^{2}=\mathbf{g}_{\vec{\rho}}(d \vec{\rho}, d \vec{\rho})=\operatorname{Tr}\left[d \vec{\rho} \mathcal{L}_{\vec{\rho}}(d \vec{\rho})\right]
$$


where $\mathbf{g}_{\vec{\rho}}$ denotes the metric tensor at point $\vec{\rho}$. Denoting $\|\mathbf{P}\| \stackrel{\text { def }}{=} m$, from (17) $d \vec{\rho}$ reads,

$$
d \vec{\rho}=\frac{1}{2} d(m \mathbf{n} \cdot \overrightarrow{\boldsymbol{\sigma}})=\frac{1}{2}(d m \mathbf{n} \cdot \overrightarrow{\boldsymbol{\sigma}}+m d \mathbf{n} \cdot \overrightarrow{\boldsymbol{\sigma}})=\frac{1}{2}(d m \mathbf{n}+m d \mathbf{n}) \cdot \overrightarrow{\boldsymbol{\sigma}},
$$

while $\mathcal{L}_{\vec{\rho}}(d \vec{\rho})$ becomes,

$$
\mathcal{L}_{\vec{\rho}}(d \vec{\rho})=\mathcal{L}_{\vec{\rho}}\left(\frac{1}{2}(d m \mathbf{n}+m d \mathbf{n}) \cdot \overrightarrow{\boldsymbol{\sigma}}\right)=\frac{d m}{2} \mathcal{L}_{\vec{\rho}}(\mathbf{n} \cdot \overrightarrow{\boldsymbol{\sigma}})+\frac{m}{2} \mathcal{L}_{\vec{\rho}}(d \mathbf{n} \cdot \overrightarrow{\boldsymbol{\sigma}}) .
$$

After some algebra, it follows that

$$
\mathcal{L}_{\vec{\rho}}(\mathbf{n} \cdot \overrightarrow{\boldsymbol{\sigma}})=\frac{2(-m \tilde{I}+\mathbf{n} \cdot \tilde{\boldsymbol{\sigma}})}{1-m^{2}} \text { and }, \mathcal{L}_{\vec{\rho}}(d \mathbf{n} \cdot \overrightarrow{\boldsymbol{\sigma}})=2 d \mathbf{n} \cdot \tilde{\boldsymbol{\sigma}}
$$

with $\tilde{\boldsymbol{\sigma}} \stackrel{\text { def }}{=}\left(\tilde{\sigma}_{1}, \tilde{\sigma}_{2}, \tilde{\sigma}_{3}\right)$. Using (20), (21) and (22) and noticing that

$$
d m \mathbf{n}+m\left(1-m^{2}\right) d \mathbf{n}=\left(1-m^{2}\right) d \boldsymbol{P}+(\mathbf{P} \cdot d \boldsymbol{P}) \mathbf{P},
$$

the line element $d s^{2}$ in (19) becomes

$$
d s^{2}=d \boldsymbol{P} \cdot d \boldsymbol{P}+\frac{(\mathbf{P} \cdot d \boldsymbol{P})^{2}}{1-m^{2}} .
$$

We now recall that if a distance between density matrices expresses statistical distinguishability then the distance must decrease under randomization (coarse-graining) [8]. Therefore, we may wonder whether or not depolarizing errors make quantum states less distinguishable by reducing their relative statistical distance. Indeed, from (16) and (24) it follows that

$$
d s^{2}=d \boldsymbol{P} \cdot d \boldsymbol{P}+\frac{1}{1-m^{2}}(\mathbf{P} \cdot d \boldsymbol{P})^{2} \rightarrow d s^{\prime 2}=d \boldsymbol{P}^{\prime} \cdot d \boldsymbol{P}^{\prime}+\frac{1}{1-m^{\prime 2}}\left(\mathbf{P}^{\prime} \cdot d \boldsymbol{P}^{\prime}\right)^{2},
$$

where $d s^{\prime 2}$ reads,

$$
d s^{\prime 2}=\left(1-\frac{4}{3} p\right)^{2} d \boldsymbol{P} \cdot d \boldsymbol{P}+\frac{\left(1-\frac{4}{3} p\right)^{4}}{\left[1-\left(1-\frac{4}{3} p\right)^{2} m^{2}\right]}(\mathbf{P} \cdot d \boldsymbol{P})^{2}
$$

Comparing (25) and (26), we observe that

$$
\left(1-\frac{4}{3} p\right)^{2} \leq 1 \text { and }, \frac{\left(1-\frac{4}{3} p\right)^{4}}{\left[1-\left(1-\frac{4}{3} p\right)^{2} m^{2}\right]} \leq \frac{1}{1-m^{2}}
$$

since $p \geq 0$ and $0 \leq m \leq 1$, respectively. Thus,

$$
d s^{2} \stackrel{\text { def }}{=}\left[d s^{2}\right]_{\text {undeformed }} \rightarrow d s^{\prime 2} \stackrel{\text { def }}{=}\left[d s^{2}\right]_{\text {depolarized }} \leq\left[d s^{2}\right]_{\text {undeformed }} \text {. }
$$

Depolarizing errors randomize quantum states rendering them less distinguishable by decreasing their relative statistical distance. Furthermore, introducing a fourth coordinate $P^{0}$,

$$
P^{0} \stackrel{\text { def }}{=} \sqrt{1-\|\mathbf{P}\|^{2}}=\sqrt{1-m^{2}}
$$

we get,

$$
d P^{0}=\frac{m^{2} d m^{2}}{1-m^{2}}=\frac{(\mathbf{P} \cdot d \boldsymbol{P})^{2}}{1-m^{2}} .
$$

Thus, the interior of the Bloch sphere is a 3 -unit sphere $\mathcal{S}^{3}$, a three-dimensional sphere of unit radius in a fourdimensional Euclidean space,

$$
\mathcal{S}^{3} \stackrel{\text { def }}{=}\left\{P^{\mu}=\left(P^{0}, P^{1}, P^{2}, P^{3}\right) \in \mathbb{R}^{4}: P^{\mu} P_{\mu}=1\right\}
$$


and the geometry on such surface is induced by the line four-dimensional flat Euclidean line element

$$
d s^{2}=d P^{\mu} d P_{\mu}=\left(d P^{0}\right)^{2}+\left(d P^{1}\right)^{2}+\left(d P^{2}\right)^{2}+\left(d P^{3}\right)^{2} .
$$

The geodesic paths for the line element $d s^{2}$ parametrized in terms of the arc-length $s$ are given by,

$$
P^{\mu}=P^{\mu}(s)=a^{\mu} \cos s+b^{\mu} \sin s
$$

where $a^{\mu}$ and $b^{\mu}$ are mutually orthogonal unit 4-vectors,

$$
a^{\mu} a_{\mu}=b^{\nu} b_{\nu}=1 \text { and, } a^{\mu} b_{\mu}=b^{\nu} a_{\nu}=0 .
$$

Trajectories in (33) are great circles, circles that have the same center and radius as the sphere. It is straightforward to show that the geodesics $P^{\prime} \mu$ on $\mathcal{S}_{\text {deformed }}^{3}$ parametrized in terms of the arc-length $s$ are given by,

$$
P^{\prime 0}(s)=\sqrt{1-\left(1-\frac{4}{3} p\right)^{2}\left[1-\left(P^{0}(s)\right)^{2}\right]} \text { and } P^{\prime k}(s)=\left(1-\frac{4}{3} p\right) P^{k}(s)
$$

for $k=1,2,3$. Thus, the three "spacial" components of the four-vector $P^{\prime} \mu$ are simply the uniformly contracted versions of the geodesic paths on $\mathcal{S}_{\text {undeformed }}^{3}$ where $1-\frac{4}{3} p$ denotes the contraction factor.

\section{COMPARISONS WITH OTHER QUANTUM DISTINGUISHABILITY METRICS}

For the sake of completeness, we point out that in the classical information geometric setting there is essentially one classical statistical distance quantifying the distinguishability between two probability distributions. Indeed, the classical Fisher information metric [9] is the only (except for an overall multiplicative constant) monotone Riemannian metric with the property of having its line element reduced under Markov morphisms (stochastic maps). In the quantum setting, Riemannian metrics are considered on the space of density matrices. The requirement that the distance between density matrices expresses quantum statistical distinguishability implies that this distance must decrease under coarse-graining (stochastic maps) [8]. Unlike the classical case, it turns out that there are infinitely many Riemannian metrics satisfying this requirement [10]. In what follows, we clarify the connections between the quantum line element we used in Section IV and other common metrics of use in the quantum framework.

\section{A. The Fubini-Study metric}

The Fubini-Study infinitesimal line element $d s_{\mathrm{FS}}^{2}$ is given by [11],

$$
d s_{\mathrm{FS}}^{2}=\|d \psi\|^{2}-|\langle\psi \mid d \psi\rangle|^{2}=1-\left|\left\langle\psi^{\prime} \mid \psi\right\rangle\right|^{2}
$$

where $|\psi\rangle$ and $\left|\psi^{\prime}\right\rangle$ are neighboring normalized pure states expanded in an orthonormal basis $\{|k\rangle\}$ with $k \in\{1, \ldots, N\}$,

$$
|\psi\rangle=\sum_{k=1}^{N} \sqrt{p_{k}} e^{i \phi_{k}}|k\rangle \text { and },\left|\psi^{\prime}\right\rangle=\sum_{k=1}^{N} \sqrt{p_{k}+d p_{k}} e^{i\left(\phi_{k}+d \phi_{k}\right)}|k\rangle,
$$

respectively. Observe that up to the second order Taylor expansion, $\left|\psi^{\prime}\right\rangle$ reads

$$
\left|\psi^{\prime}\right\rangle=\sum_{k=1}^{N}\left[\sqrt{p_{k}}\left(1+\frac{1}{2} \frac{d p_{k}}{p_{k}}-\frac{1}{8} \frac{d p_{k}^{2}}{p_{k}^{2}}\right) e^{i \phi_{k}}\left(1+i d \phi_{k}-\frac{1}{2} d \phi_{k}^{2}\right)\right]|k\rangle .
$$

Upon use of the normalization constraint and its differential form, $\sum_{k=1}^{N} p_{k}=1$ and $\sum_{k=1}^{N} d p_{k}=0$ respectively, $\left\langle\psi^{\prime} \mid \psi\right\rangle$ becomes

$$
\left\langle\psi^{\prime} \mid \psi\right\rangle=1-\frac{1}{8} \sum_{k=1}^{N} \frac{d p_{k}^{2}}{p_{k}}-i \sum_{k=1}^{N} p_{k} d \phi_{k}-\frac{i}{2} \sum_{k=1}^{N} d p_{k} d \phi_{k}-\frac{1}{2} \sum_{k=1}^{N} p_{k} d \phi_{k}^{2}
$$


It is straightforward to compute $\left|\left\langle\psi^{\prime} \mid \psi\right\rangle\right|^{2}$ and to arrive at the Fubini-Study infinitesimal line element

$$
d s_{\mathrm{FS}}^{2}=\frac{1}{4} \sum_{k=1}^{N} \frac{d p_{k}^{2}}{p_{k}}+\sum_{k=1}^{N} p_{k} d \phi_{k}^{2}-\left(\sum_{k=1}^{N} p_{k} d \phi_{k}\right)^{2} .
$$

It is also $d s_{\mathrm{FS}}^{2}=\left\langle d \psi_{\perp} \mid d \psi_{\perp}\right\rangle$, where $\left|d \psi_{\perp}\right\rangle \stackrel{\text { def }}{=}|d \psi\rangle-\langle\psi \mid d \psi\rangle|\psi\rangle$ is the projection of $|d \psi\rangle$ orthogonal to $|\psi\rangle$ with $|d \psi\rangle \stackrel{\text { def }}{=}\left|\psi^{\prime}\right\rangle-|\psi\rangle$. Then, for pure states $\rho=|\psi\rangle\langle\psi|$ and $d \rho=|\psi\rangle\left\langle d \psi_{\perp}|+| d \psi_{\perp}\right\rangle\langle\psi|$, Eq. (11) reduces to

$$
d s^{2}=2 \operatorname{tr}\left(d \rho^{2}\right)=4\left\langle d \psi_{\perp} \mid d \psi_{\perp}\right\rangle,
$$

which is, except for an overall real constant, the Fubini-Study metric (40).

In conclusion, due to the fact that the Fubini-Study metric quantifies distinguishability of pure states only, it is not a useful metric for a geometric characterization of depolarizing channels.

\section{B. The Bures metric}

For a detailed presentation concerning the computation of the Bures metric for two-dimensional density matrices, we refer to [12]. Consider two density matrices $\rho_{1}$ and $\rho_{2}$,

$$
\rho_{1}=\frac{1}{2} I+\mathbf{x}_{1} \cdot \boldsymbol{\sigma} \text { and, } \rho_{2}=\frac{1}{2} I+\mathbf{x}_{2} \cdot \boldsymbol{\sigma}
$$

with $\mathbf{x}_{i}$ in $\mathbb{R}^{3}$ and $\boldsymbol{\sigma}=\left(\sigma_{x}, \sigma_{y}, \sigma_{z}\right)$. By definition, $\rho_{1}$ and $\rho_{2}$ must have positive eigenvalues and this implies that the magnitude of $\mathbf{x}_{k}$ with $k=1,2$ is less than or equal to one-half. We stress that the operator-representation (42) differs from Eq.(17) we used in our analysis (in that case, the magnitude of $\mathbf{x}_{k}$ was less or equal to one).

For the sake of reasoning, consider two unphysical $\left(\operatorname{tr} \rho_{k} \neq 1\right)$ density matrices given by, $\rho_{1}=\alpha_{1} I+\mathbf{x}_{1} \cdot \boldsymbol{\sigma}$ and, $\rho_{2}=$ $\alpha_{2} I+\mathbf{x}_{2} \cdot \boldsymbol{\sigma}$, where $\alpha_{k}^{2} \stackrel{\text { def }}{=} \mathbf{x}_{k}^{2}+b_{k}^{2}$ ad, $b_{k} \stackrel{\text { def }}{=} \sqrt{\operatorname{det} \rho_{k}}$. Then, the Bures distance between $\rho_{1}$ and $\rho_{2}$ reads [12],

$$
d_{\text {Bures }}^{2}\left(\rho_{1}, \rho_{2}\right)=2\left(\alpha_{1}+\alpha_{2}\right)-2^{\frac{3}{2}} \sqrt{\alpha_{1} \alpha_{2}+\mathbf{x}_{1} \cdot \mathbf{x}_{2}+b_{1} b_{2}} .
$$

To connect the Bures distance in (43) to our quantum line element (24), we have to take into consideration two density $\rho_{1}$ and $\rho_{2}$ matrices infinitesimally near to each other. Assume

$$
\rho_{1}=\frac{1}{2} I+\mathbf{x} \cdot \boldsymbol{\sigma} \text { and } \rho_{2}=\rho_{1}+d \rho .
$$

with $d \rho \stackrel{\text { def }}{=} \mathbf{d x} \cdot \boldsymbol{\sigma}$. Then, it turns out that the second order expansion of the Bures distance Eq. (43) with $\rho_{1}$ and $\rho_{2}$ given in Eq.(44) reads $d s_{\text {Bures }}^{2}=\frac{1}{2} \operatorname{tr}\left[(d \rho)^{2}\right]+(d \sqrt{\operatorname{det} \rho})^{2}$, that is

$$
d s_{\text {Bures }}^{2}=\mathbf{d x} \cdot \mathbf{d x}+d b d b=\mathbf{d x} \cdot \mathbf{d x}+\frac{(\mathbf{x} \cdot \mathbf{d x})^{2}}{\frac{1}{4}-\mathbf{x}^{2}},
$$

where the four coordinates $\mathbf{x}$ and $b$ satisfy the normalization condition $\mathbf{x}^{2}+b^{2}=1 / 4$. Here they come further differences with our analysis carried out in Section IV: our four coordinates are normalized to one. Furthermore, while the set of two-dimensional normalized density matrices equipped with the Bures metric is isometric to one closed half of the three-sphere with radius $\frac{1}{2}$, with our quantum line metric (24) the set of density matrices is isometric to one closed half of the three-sphere with radius 1.

It is straightforward to check that regardless of the chosen metric (our metric (24) or that of Bures (45)), the geometric characterization of the depolarizing channel does not change in its substance. Loosely speaking, the only basic difference is that in the Bures case, we consider deformation properties on a sphere of radius $\frac{1}{2}$ instead of 1 . In summary, it can be shown that,

$$
\left[d s_{\text {Bures }}^{2}\right]_{\text {depolarized }} \leq\left[d s_{\text {Bures }}^{2}\right]_{\text {not-deformed }} \Leftrightarrow \mathbf{x}^{2} \leq \frac{1}{4}\left[1+\frac{1}{\left(1-\frac{4}{3} p\right)^{2}}\right],
$$

which is true since the magnitude of $\mathbf{x}$ is less or equal to one-half and where $p \in[0,1]$ denotes the error probability. 


\section{FINAL REMARKS}

Relying on the possibility of introducing a Riemannian geometric structure on the space of density operators based on the statistical concept of distinguishability, we investigated the conceptual usefulness of differential geometric tools in quantifying the effect of a noisy depolarizing channel on quantum states by comparing the geometries of the interior of the undeformed and depolarized Bloch spheres related to density operators on a two-dimensional Hilbert space. In particular, we have pointed out that the two main features that characterize the action of the depolarizing channel on density operators, namely the randomization of maximally entangled quantum states (Eq. (14)) and the uniform contraction of the deformed Bloch sphere (Eq. (16)) can be both quantified in differential geometric terms. Randomization emerges geometrically through a smaller infinitesimal quantum line element on the deformed Bloch sphere (Eq. (28) ) while the uniform contraction manifests itself via a deformed set of geodesics where the spacial components of the deformed four-Bloch vector are simply the contracted versions of the undeformed Bloch vector components (Eq. (35)).

In agreement with [13], we believe that this preliminary analysis deserves further investigation especially in regard to a possible differential geometric quantification of quantum randomness in measurement theory related to a physical characterization of the Kraus operator-sum decomposition of arbitrary quantum noisy communication channels.

\section{Acknowledgments}

The Authors thank Giuseppe Marmo for his kind hospitality and for very enlightening discussions during their short visit at the Università di Napoli "Federico II".

[1] T. Frankel, "The Geometry of Physics", Cambridge University Press (1997).

[2] G. Marmo and G. F. Volkert, "Geometrical description of quantum mechanics-transformations and dynamics", Phys. Scr. 82, 038117 (2010).

[3] L. L. Campbell, "The Relation Between Information Theory and the Differential Geometry Approach to Statistics", Inform. Sci. 35, 199 (1985).

[4] S. L. Braunstein and C. M. Caves, "Geometry of Quantum States", Annals of the New York Academy of Sciences 755, 786 (1995).

[5] C. A. Fuchs, "Distinguishability and Accessible Information in Quantum Theory", PhD Thesis, University of New Mexico, USA (1995).

[6] S. L. Braunstein and C. M. Caves, "Statistical Distance and the Geometry of Quantum States", Phys. Rev. Lett. 72, 3439 (1994).

[7] J. Preskill, "Lecture Notes for Physics 229: Quantum Information and Computation", California Institute of Technology, USA (1998).

[8] D. Petz and C. Sudar, "Geometries of quantum states", J. Math. Phys. 37, 2662 (1996).

[9] L. L. Campbell, "An extended Cencov characterization of the information metric", Proc. Am. Math. Soc. 98, 135 (1986).

[10] D. Petz, "Monotone Metrics on Matrix Spaces", Lin. Alg. Appl. 244, 81 (1996).

[11] J. P. Provost and G. Vallee, "Riemannian Structure on Manifolds of Quantum States", Comm. Math. Phys. 76, 289 (1980).

[12] M. Hubner, "Explicit computation of the Bures distance for density matrices", Phys. Lett. A 163, 239 (1992).

[13] V. I. Man'ko, G. Marmo, E. C. G. Sudarshan and F. Zaccaria, "Differential geometry of density states", Rep. Math. Phys. $\mathbf{5 5}, 405$ (2005). 